\title{
THE EFFECTIVENESS OF IMPLEMENTING LEAN MANUFACTURING TECHNIQUES
}

\author{
Ananthukrishna.A \\ Ex. General Manager, Sree Vivekananda Padana Kendram Foundation, \\ Malappuram, Kerala, India
}

\begin{abstract}
Lean manufacturing is a set of techniques and tools for process improvement. It was developed by Motorola in 1986, coinciding with the Japanese asset price bubble which is reflected in its terminology. Jack Welch made it central to his business strategy at General Electric in 1995. Today, it is used in many industrial sectors.

The term "lean manufacturing" comes from statistics and is used in statistical quality control, which evaluates process capability. Originally, it referred to the ability of manufacturing processes to produce a very high proportion of output within specification. Processes that operate with "lean manufacturing quality" over the short term are assumed to produce long-term defect levels below 3.4 defects per million opportunities (DPMO).

The primary objective is to study the effectiveness of implementing lean manufacturing techniques and to identify and analyze any related issues and industrial common challenges or problems in lean manufacturing implementation. In this study Descriptive Research design is used.

In the case of multiple choice questions the responses are categorized based on the nature and percentage, and is calculated for each category. To find the percentage of the responses in each category Percentage Analysis is used. The percentage is calculated by dividing the number of responses by the total number of respondents.

Convenience sampling method has been used in the research work. A wellstructured questionnaire have been used to collect the responses from employees. Numerous findings has been derived from this research has helped to provide few suggestions to improve the effectiveness of lean manufacturing techniques.
\end{abstract}

Keywords: lean manufacturing, manufacturing techniques

Cite this Article: Ananthukrishna.A, the Effectiveness of Implementing Lean Manufacturing Techniques, International Journal of Management, 10 (2), 2019, pp. 47-51.

http://www.iaeme.com/IJM/issues.asp?JType=IJM\&VType=10\&IType=2

\section{INTRODUCTION}

The term Lean manufacturing originated from terminology associated with manufacturing, specifically terms associated with statistical modelling of manufacturing processes. The 
maturity of a manufacturing process can be described by a sigma rating indicating its yield or the percentage of defect-free products it creates. A lean manufacturing process is one in which $99.99966 \%$ of the products manufactured are statistically expected to be free of defects (3.4 defective parts/million), although, as discussed below, this defect level corresponds to only a 4.5 sigma level. Motorola set a goal of "lean manufacturing" for all of its manufacturing operations, and this goal became a by-word for the management and engineering practices used to achieve it.

Most of the basic goals of lean manufacturing and waste reduction were derived from Benjamin Franklin through documented examples. Poor Richard's Almanack says of wasted time, "He that idly loses $5 \mathrm{~s}$. worth of time, loses $5 \mathrm{~s}$., and might as prudently throw $5 \mathrm{~s}$. into the river." He added that avoiding unnecessary costs could be more profitable than increasing sales: "A penny saved is two pence clear. A pin a-day is a groat a-year. Save and have."

Frederick Winslow Taylor, the father of scientific management, introduced what are now called standardization and best practice deployment. In Principles of Scientific Management, (1911), Taylor said: "And whenever a workman proposes an improvement, it should be the policy of the management to make a careful analysis of the new method, and if necessary conduct a series of experiments to determine accurately the relative merit of the new suggestion and of the old standard. And whenever the new method is found to be markedly superior to the old, it should be adopted as the standard for the whole establishment."

Ford's success has startled the country, almost the world, financially, industrially, mechanically. It exhibits in higher degree than most persons would have thought possible the seemingly contradictory requirements of true efficiency, which are: constant increase of quality, great increase of pay to the workers, repeated reduction in cost to the consumer. And with these appears, as at once cause and effect, an absolutely incredible enlargement of output reaching something like one hundredfold in less than ten years, and an enormous profit to the manufacturer.

Lean principles have been successfully applied to various sectors and services, such as call centers and healthcare. In the former, lean's waste reduction practices have been used to reduce handle time, within and between agent variation, accent barriers, as well as attain near perfect process adherence. In the latter, several hospitals have adopted the idea of lean hospital, a concept that priorizes the patient, thus increasing the employee commitment and motivation, as well as boosting medical quality and cost effectiveness.

The wire and cable industry has moved from being a small industry 20 years back to a very large industry over the last decade. Although it is a volume-driven product, it has a lot of quality and technical particulars. Over a period of time, the industry has moved from the unorganized sector. However, about $35 \%$ of this industry is still in the unorganized sector.

\section{THE ISSUE OF CULTURE AND FIVE CULTURAL CHANGE LEADING INDICATORS}

The culture is defined as "the combined action, thoughts, values, beliefs, artifacts, and language of any group of people." There are five reasons that make it worthwhile to consciously create and sustain a healthy culture. First, goal alignment is much easier, second, workers are more highly motivated in a predictable culture and will not only take action with more confidence, there will also be more likely to initiate new methods.

The three major contributors to the culture of any business are: the external business environment, the few key managers at the top, and the history of business. Most important is the business environment, this is the largest driving factor. The area of the country you work in, the labour market, the products you make, the degree of government control, and a litany of other external factors all impact your culture. 


\section{LEADERSHIP}

To act properly, the leader must first be able to recognize exactly what the situation is, and they must be accurately aware of happenings in the facility. Second, they themselves must is excellent problem solvers. They must be able to sort through the options and properly apply the values needed. Finally, they must have the wisdom, courage and character to act when actions is required, and conversely should use those same skills to hold back when thoughtful inaction is the appropriate behavior. So leader of lean transformation needs ;

- The ability to develop a vision- based plan

- The ability to articulate this plan and engage others

- The ability to act on the plan

\section{MOTIVATION}

The changes in lean transformation are much like the changes must undertake,

- Have a clear goal in sight.

- Recognize that we need to change to reach the goal

- Recognize that the changes will be uncomfortable and even painful at times

- Recognize that the short term problems and losses will be just that, short term; and we must keep our eye on the target, the long term objectives.

- Recognize there will be forces within ourselves and outside ourselves, which are driven by different motivations. These forces will resist the necessary changes and often coax us in wrong direction.

\section{PROBLEM SOLVING}

The basic principles of problem solving and mantra is " think small, think fast, think lots" of cycles through rapid response PDCA( Plan, Do, Check, Act) process is important.

The problem solvers who have requisite skills to solve problems are rare indeed. They must be able to ; exhibit great observation skills and readily grasp the situation; turn it into a meaningful problem statement; know how to gather and sort through the data; Analyze the data and information, including doing a statistical review; utilize root-cause problem analysis; create a list of possible solutions; sort; decide

\subsection{FACILITY ENGAGEMENT}

Facility engagement knows what to do to accomplish the task at hand, knows how to do the job, has the resources to do the job, wants to do the job and wants to do it better.

\subsection{LEARNING, TEACHING, EXPERIMENTING ENVIRONMENT}

To explain many of the aspects of this critical cultural change leading indicator that is almost always minimized and frequently even over looked.

- Know the purpose of the data

- Collect it efficiently

- Take action based on the data

\section{LITERATURE REVIEW}

Jamal Ahmed Hama Kareem, Pirshing Salih Mohamad Al Askari1 and Farooq Hussain Muhammad, (2017) in their study entitled "Critical issues in Lean Manufacturing" studied to investigate the critical issues affecting the success of implementing lean manufacturing programs in the iron and steel factories in the Kurdistan Region of Iraq. To achieve this end, the mixed methods represented in a questionnaire survey and semi-structured interviews to 
collect the data in the framework of the case study were chosen. Based on the findings, the organization managements are recommended to provide financial and moral support to employees to enable a successful implementation of lean manufacturing aimed at obtaining the desired results of them.

Nurul Husna Zakaria, Nik Mohd Zuki Nik Mohamed, Mohd Fadzil Faisae Ab Rahid and Ahmad Nasser Mohd Rose, (2017) in the study entitled "Lean manufacturing implementation in reducing waste for electronic assembly line" studied to investigate possibilities and sustainability of application of lean manufacturing method by using a virtual simulation of the workers performance in a line production of small and medium industry. Lean manufacturing method has been used during the study to reduce the cost when waste is eliminated by reducing the workstation without reducing the performance of the production. The performance of the production is increased when allocating the labor in a needed working area. Lastly, the study also proves that the new layout has improved the process to be used for future production process. The virtual simulation was simulated based on data collections gained during observation process. According to the results obtained, the waste that had been addressed by this simulation is processing waste; a situation of a particular process step that does not contribute any benefit to the product where the two labours in one process are having a small percent of busy.

Da Silva de Santis SH, Dedini FG, Marcicano JPP and de Santis , (2016) undergone study on the topic called "Lean Manufacturing and Value Engineering, Two Concepts for Sustainable Management" studied to evaluate the lean production concepts usage in the textile industry. The goal is to investigate the management practices that contribute to the development of sustainable production processes. The adjustments necessary to the system development focuses on the management of resources and inputs of production processes to promote the best use of these and still cutting costs, making the company sustainable economy. The purpose, therefore, is to deploy management practices, tools and routines that help in the company's processes. The research will be developed through case study, we intend to analyse the reality of the organization. The data collected through interviews, questionnaires and secondary source (books, articles and magazines) that will be used as the basis of information and provide the necessary clarification for the rationale. It was observed that several authors advocate the use of these concepts in management. Comparing the two concepts that either a lot of similarity and was said to complement each other when used for process optimization.

Srinivasa Rao P, Malay Niraj, (2016) in the study entitled "A case study on implementing lean ergonomic manufacturing systems (LEMS) in an automobile industry". The study demonstrates the adoption of ergonomic conditions in lean manufacturing for the improvement of organizational performance of the industry. The aim of ergonomics is to adapt the new techniques to their work in efficient and safe ways in order to optimize the human health conditions and increasing the production rate. By conducting survey on various disciplines and showed how the production rate and human ergonomic conditions is affected. The model of a framework regarding the integration of Ergonomics and lean manufacturing systems based on the various tools was presented. The proposed framework associates to the lean manufacturing system procedures used in each phase of the ergonomic tools and methodologies introducing an additional ergonomic perspective.

R. Coetzee, K. Van Der Merwe, L.Van Dyk, (2016) in their study entitled "Lean Implementation Strategies: How are the Toyota way Principles Addressed?" studied the extent to which the human dimensions of the lean philosophy, as described in the Toyota Way management principles, have been incorporated in lean implementation strategies. The adoption of the lean philosophy runs contrary to the oft-repeated message from its creators that 
no tenet of the philosophy should be favoured at the expense of another. This may provide valuable insight into the reasons for the high implementation failure rate.

S.Gobinath, D.Elangovan, S.Dharmalingam, (2015) in their study entitled "Lean Manufacturing Issues and Challenges in Manufacturing process" studied one of the major difficulties companies encounter in attempting to apply lean is not knowledge of particular tools and techniques perhaps lack of comprehensive and suitable lean knowledge related probable problems within the companies by the managers, direction, gap and lack of recognition of lean culture in whole of the organization and planning cause the fails within the implementation.Lack of planning, lack of top management commitment, lack of methodology, unwillingness to learn and see and human aspects are the main barriers or problems which can be faced while implementing the lean manufacturing.

\section{CONCLUSION}

Today, Lean manufacturing is being utilized by many business firms of all sizes. Although it took a few years to become main stream, the success stories from the middle companies to large companies pushed hard the small companies to adopt it for the better performance and reduction in waste and non-value added operations. Most of the large companies employ a few lean manufacturing experts. Many medium and small companies do not have a lean expertise in the company. but it is difficult to develop a roadmap for smooth conducting of the process. Lean manufacturing is a systematic method for waste minimization within a manufacturing system without sacrificing productivity. Lean also taken into account that waste created through overburden and waste created through unevenness in workloads.

This study mainly reveals the effectiveness of implementing lean manufacturing techniques. This study provides the benefits of lean manufacturing and its effectiveness on day to day operations of the organization.

\section{REFERENCES}

[1] Critical issues in Lean Manufacturing by Jamal Ahmed Hama Kareem, Pirshing Salih Mohamad Al Askari1 and Farooq Hussain Muhammad.

[2] Lean manufacturing implementation in reducing waste for electronic assembly line by Nurul Husna Zakaria, Nik Mohd Zuki Nik Mohamed, Mohd Fadzil Faisae Ab Rahid and Ahmad Nasser Mohd Rose.

[3] Lean Manufacturing and Value Engineering, Two Concepts for Sustainable Management by Da Silva de Santis SH, Dedini FG, Marcicano JPP and de Santis.

[4] A case study on implementing lean ergonomic manufacturing systems (LEMS) in an automobile industry by Srinivasa Rao P, Malay Niraj.

[5] Lean Implementation Strategies: How are the Toyota way Principles Addressed by R. Coetzee, K. Van Der Merwe, L.Van Dyk.

[6] Lean Manufacturing Issues and Challenges in Manufacturing process by S.Gobinath, D.Elangovan, S.Dharmalingam.

[7] Green- Lean synergy- Root-Cause Analysis in food waste prevention by Regah Amani, Ingela Lindbom, Barbro Sundstrom and Karin Ostergren.

[8] Lean Manufacturing Tools Implementation and Its Impact on the Company's Operation Improvement by Grazyna Paulina Wojcik, Klaudia Kocon. 